5. Liu B, et al. The acute nociceptive signals induced by bradykinin in rat sensory neurons are mediated by inhibition of $\mathrm{M}$-type $\mathrm{K}^{+}$channels and activation of $\mathrm{Ca}^{2+}$-actived $\mathrm{Cl}^{-}$channels. J Clin Invest. 2010;120(4):1240-1252.

6. Passmore GM, et al. KCNQ/M currents in sensory neurons: significance for pain therapy. J Neurosci. 2003;23(18):7227-7236.

7. Gamper N, Shapiro MS. Calmodulin mediates $\mathrm{Ca}^{2+}$-dependent modulation of $\mathrm{M}$-type $\mathrm{K}^{+}$channels. J Gen Physiol. 2003;122(1):17-31.

8. Jones $\mathrm{S}$, et al. Bradykinin excites rat sympathetic neurons by inhibition of $\mathrm{M}$ current through a mechanism involving $\mathrm{B} 2$ receptors and $\mathrm{G}$ alpha $\mathrm{q} / 11$. Neuron. 1995;14(2):399-405.

9. Adams PR, Brown DA. Actions of gamma-aminobutyric acid on sympathetic ganglion cells. J Physiol. 1975;250(1):85-120.

10. Yang YD, et al. TMEM16A confers receptor-activated calcium-dependent chloride conductance. Nature. 2008;455(7217):1210-1215.

11. Caputo A, et al. TMEM16A, a membrane protein associated with calcium-dependent chloride channel activity. Science. 2008;322(5901):590-594

12. Schroeder BC, Cheng T, Jan YN, Jan LY. Expression cloning of TMEM16A as a calcium-acti- vated chloride channel subunit. Cell. 2008; 134(6):1019-1029.

13. Passmore GM, Brown DA. Effects of M-channel modulators on peripheral excitability in rat hairy skin. Program no. 681.8. 2007 Neuroscience Meeting Planner. San Diego, California, USA: Society for Neuroscience.

14. Roza C, Lopez-Garcia JA. Retigabine, the specific KCNQ channel opener, blocks ectopic discharges in axotomized sensory fibres. Pain. 2008;138(3):537-545.

15. Ault B, Hildebrand LM. GABA, Receptor-mediated excitation of nociceptive afferents in the rat isolated spinal cord-tail preparation. Neuropharmacology. 1994;33(1):109-114.

16. Higashida H, Brown DA. Two polyphosphoinositide metabolites control two $\mathrm{K}^{+}$currents in a neuronal cell. Nature. 1986;323(6086):333-335.

17 Funk K, et al. Modulation of chloride homeostasis by inflammatory mediators in dorsal root ganglion neurons. Mol Pain. 2008;4:32.

18. Wickenden AD, McNaughton-Smith G. Kv7 Channels as targets for the treatment of pain. Curr Pharm Des. 2009;15(15):1773-1789.

19. Lang PM, Fleckenstein J, Passmore GM, Brown DA, Grafe P. Retigabine reduces the excitability of unmyelinated peripheral human axons. Neurophar- macology. 2008;54(8):1271-1278.

20. Ferreira J, et al. Reduced nerve injury-induced neuropathic pain in kinin B1 receptor knock-out mice. JNeurosci. 2005;25(9):2405-2412.

21. Patwardhan AM, et al. Heat generates oxidized linoleic acid metabolites that activate TRPV1 and produce pain in rodents. J Clin Invest. 2010;120(5):1617-1626.

22. Tominaga $\mathrm{M}$, et al. The cloned capsaicin receptor integrates multiple pain-producing stimuli. Neuron. 1998;21(3):531-543.

23. Patwardhan AM, Scotland PE, Akopian AN, Hargreaves KM. Activation of TRPV1 in the spinal cord by oxidized linoleic acid metabolites contributes to inflammatory hyperalgesia. Proc Natl Acad SciU S A. 2009;106(44):18820-18824.

24. Lukacs V, Thyagarajan B, Varnai P, Balla A, Balla $\mathrm{T}$, Rohacs T. Dual regulation of TRPV1 by phosphoinositides. J Neurosci. 2007;27(26):7070-7080.

25. Gamper N, Shapiro MS. Regulation of ion transport proteins by membrane phosphoinositides. Nat Rev Neurosci. 2007;8(12):921-934.

26. Voets T, Droogmans G, Wissenbach U, Janssens A, Flockerzi V, Nilius B. The principle of temperaturedependent gating in cold- and heat-sensitive TRP channels. Nature. 2004;430(7001):748-754.

\title{
Neuronal injury: folate to the rescue?
}

\section{Golo Kronenberg ${ }^{1,2,3}$ and Matthias Endres 1,2}

\begin{abstract}
${ }^{1}$ Klinik und Poliklinik für Neurologie and ${ }^{2}$ Center for Stroke Research Berlin, Charité - Universitätsmedizin Berlin, Berlin, Germany.
${ }^{3}$ Klinik und Hochschulambulanz für Psychiatrie und Psychotherapie, Charité - Universitätsmedizin Berlin, Campus Benjamin Franklin, Berlin, Germany.
\end{abstract}

\begin{abstract}
Strong epidemiological evidence indicates that derangement of single-carbon metabolism has detrimental effects for proper CNS functioning. Conversely, a role for folate supplementation in the treatment and prevention of neurodegenerative and neuropsychiatric disorders remains to be established. In this issue of the JCI, in an elegant series of experiments in rodents, Iskandar and colleagues demonstrate a crucial role of folate in the regeneration of afferent spinal neurons after injury. Probing sequential steps in folate metabolism, from cellular entry to DNA methylation, the authors show that axonal regeneration relies upon the integrity of DNA methylation pathways. These findings provide the first demonstration of an epigenetic mechanism contributing to neurorepair and suggest that manipulation of the methylation milieu may offer promising new therapeutic avenues to promote regeneration.
\end{abstract}

Folic acid, also referred to as folate (the anion form) or vitamin B9, plays a central role in single-carbon metabolism. Specifically, in a reaction dependent on vitamin B12, N5-methyltetrahydrofolate acts as a one-carbon donor in the remethylation of homocysteine to methionine. Dietary intake of methionine as such is insufficient to provide enough methyl groups that are required for cellular methylation reactions. Therefore, the body has

Conflict of interest: The authors have declared that no conflict of interest exists.

Citation for this article: $J$ Clin Invest. 2010; 120(5):1383-1386. doi:10.1172/JCI40764. to rely on the folate-dependent de novo generation of methyl groups via the single-carbon pool. Methionine is further converted to S-adenosylmethionine (SAM), which was discovered in 1953 as the "active methionine" by Catoni (1). The methyl moiety attached to the sulfur atom in SAM is chemically reactive. This explains why SAM is able to serve as the principal methyl donor in most biological transmethylation reactions (e.g., methylation of nucleic acids, proteins, or lipids). The transfer of the methyl group of SAM to an acceptor yields S-adenosylhomocysteine (SAH). Dietary folate deficiency therefore confers a strong decrease in the ratio of SAM to SAH. The ratio of these two compounds regulates the balance of many cellular methylation reactions, not least that of genomic DNA. Accordingly, DNA hypomethylation has been demonstrated experimentally as a consequence of dietary folate deficiency (2).

As a water-soluble vitamin, folate cannot be stored in large amounts in the body. The special importance of folate for CNS functioning is evidenced by the fact that in deficiency states, the brain is relatively spared from folate deficiency as compared with other tissues (3). Uptake of folate across the blood-CSF barrier is primarily accomplished via membranebound folate receptors. Autoantibodies against these receptors may lead to cerebral folate deficiency syndrome, a pediatric neurological disorder characterized by psychomotor retardation, cerebellar ataxia, seizures, and low CSF levels of methyltetrahydrofolate (4). By now, solid evidence has also been assembled that establishes an association between folate metabolism and neural tube defects (5), although underlying mechanisms have not been fully elucidated. Furthermore, low folate status and hyperhomocysteinemia have been implicated as poten- 


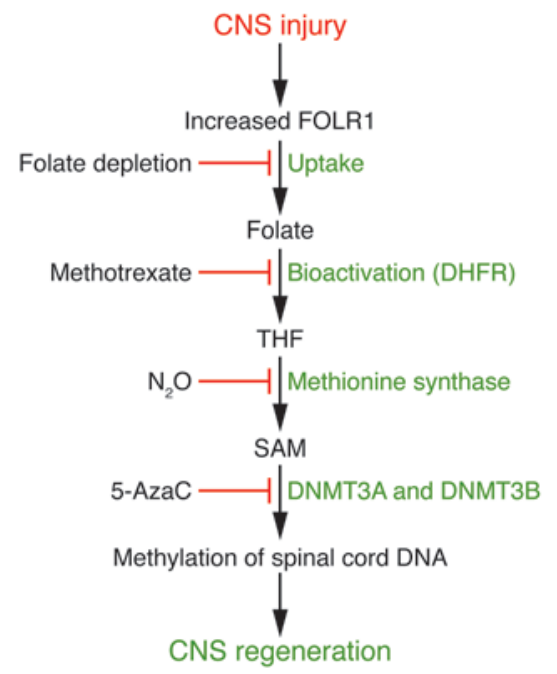

Figure 1

Role of folate in axonal regeneration. The figure summarizes the sequence of events reported in this issue of the $\mathrm{JCl}$ by Iskandar et al. (7) that underlie the proregenerative effects of folate on injured CNS tissue in their rodent models. Experimental manipulations that can block this pathway at specific steps are shown on the left. Following transection injury to the CNS, increased expression of FOLR1 promotes folate uptake. Folate is then reduced to tetrahydrofolate (THF) by DHFR. Methyl-THF is required for the regeneration of SAM, which in turn promotes methylation of spinal cord DNA. Sufficient DNA methylation is crucial for axonal regeneration. 5-AzaC, 5-azacytidine.

tially reversible risk factors for cerebrovascular disease, cognitive decline in the elderly, Alzheimer disease, and depression (reviewed in ref. 6).

In this issue of the JCI, Iskandar and colleagues link folate status to an epigenetic mechanism of CNS de- and regeneration in rodents (7). An epigenetic trait has been defined as "a stably inherited phenotype resulting from changes in a chromosome without alterations in the DNA sequence" (8). DNA methylation is a major epigenetic mechanism central to regulation of gene transcription, chromatin structure, imprinting, development, and genomic stability in general. In higher organisms, a methyl moiety is preferentially, albeit not exclusively, targeted to the DNA base cytosine in the context of a CPG dinucleotide. In mammals, DNA is highly methylated in $\mathrm{CpG}$ sequences (>50\%). DNA methylation is associated with a condensed structure and transcriptional repression. It is believed to have originated as a host defense mechanism for silencing para- sitic DNA elements such as transposons and retrotransposons. The agouti mouse model provides a fascinating demonstration of the importance of methyl donors, including folic acid, in development: early methyl donor nutrition during pregnancy causes methylation and thereby silencing of a retrotransposon, with silencing of the nearby agouti gene altering fur color in the offspring (9). DNA methylation is catalyzed by DNA nucleotide methyltransferases (DNMTs). DNMT1 serves as a "maintenance" methyltransferase and accordingly shows 30 - to 40 -fold preference for hemimethylated sites (10). In contrast, DNMT3A and DNMT3B serve as de novo methyltransferases.

Altered patterns of DNA methylation, in particular hypomethylation of growthpromoting genes and hypermethylation of tumor suppressor genes, have become a common theme in cancer biology. Additionally, several single-gene disorders of the epigenetic machinery have been uncovered. Rett syndrome is a neurodevelopmental disorder caused by mutations of methyl CpG-binding protein 2 (MeCP2), which regulates the expression of a wide variety of genes (11). Immunodeficiency/ centromeric instability/facial anomalies (ICF) syndrome involves mutations in DNMT3B and is also associated with variable degrees of mental retardation (12). Another striking example of an epigenetic mechanism operating in the brain is the effect of maternal behavior on DNA methylation at a glucocorticoid receptor gene promoter in the hippocampus of rat pups (13). Considering that epigenetics is central to phenotypic variation, understanding and modifying the epigenome holds great promise for the treatment and prevention of human disease.

\section{Spinal cord injury, the folate pathway, and DNA methylation}

Iskandar et al. investigated the consequences of sharp transection injuries on folate metabolism in rodents (7). Figure 1 provides an overview of the major experimental results. To sever primary somatosensory axons, the cervical dorsal column was lesioned. Additionally, transection of the sciatic nerve corresponded to injury of peripheral axons. Four days after injury, the authors found a 2 -fold increase in the percentage of cells expressing the $\alpha$-isoform of folate receptor 1 (FOLR1) and a 6 -fold increase in the percentage of cells immunostaining for FOLR1. Interesting- ly, low extracellular folate concentration has been shown to induce folate receptor expression in human cultured cells (14). The folate receptor is a polypeptide that binds folate with high affinity. In contrast, the reduced folate carrier (RFC) is characterized by a significantly lower affinity for folic acid (15). Iskandar et al. found minimal change in $R f c 1$ mRNA expression and protein levels in the injured animals as compared with uninjured controls (7). Three days after cord injury, the SAM/SAH ratio (see above) had declined by approximately $50 \%$. Consistent with the rise in SAH, a methionine synthase (MS) activity assay of spinal cord after transection injury revealed reduced enzymatic activity, whereas MS protein levels observed on Western blots did not change. Following spinal cord injury, no effects on DNMT1 protein levels were observed. However, DNMT3A and DNMT3B protein levels were strongly suppressed after cord injury. Finally, three days after sharp injury, global DNA methylation was significantly decreased.

Next, the authors assessed the therapeutic effects of folate in the spinal cord and optic pathways (7). After transection injury, an autologous sciatic nerve graft was implanted at the injury site (i.e., spinal cord or optic stump, respectively). The other end of the graft was allowed to lie freely under the skin. After an interval of time sufficient to allow growth of axons into the graft, the end of the graft was filled with the retrograde neuronal tracer hydroxystilbamidine (Fluoro-Gold). Fluorescently labeled cells were then quantified in the dorsal root ganglia (DRG) or retina, respectively. Folate supplementation of wild-type animals produced a strong increase in the percentage of regenerating spinal neurons and of regenerating retinal ganglion cells relative to wildtype animals without folate treatment. This proregenerative effect of folic acid was abrogated in $\mathrm{Folr}^{+/-}$heterozygous mice, suggesting that increased expression of FOLR1 subsequent to axonal damage is critical to folate-mediated neuroregeneration.

Dihydrofolate reductase (DHFR) catalyzes the reduction of folate to dihydrofolate and then further to tetrahydrofolate. To determine whether the propitious effects of folate depended on its initial bioactivation, Iskandar and colleagues repeated the optic nerve and spinal cord lesion experiments in animals pretreated 
with DHFR antagonist methotrexate (MTX) (7). Again, DHFR inhibition abrogated the proregenerative effects of folate. In addition, the authors used nitrous oxide $\left(\mathrm{N}_{2} \mathrm{O}\right)$, which irreversibly inactivates MS, to determine whether the integrity of the single-carbon cycle is necessary for the beneficial effects of folate. Again, in animals breathing $\mathrm{N}_{2} \mathrm{O}$, neuronal regeneration decreased to baseline levels in both injury models. Finally, the authors demonstrated that after spinal cord injury, folic acid treatment restored de novo DNMT3A and DNMT3B protein expression. Importantly, DNMT agonism promoted, and DNMT antagonism inhibited, growth of injured sensory spinal axons into the nerve graft.

Interestingly, the authors found an inverted U-shaped relationship between the therapeutic effect of folic acid and the dose administered, with peak therapeutic effects observed at $80 \mu \mathrm{g} / \mathrm{kg}$ (7). A similar biphasic increase and subsequent decrease in spinal cord global methylation was observed in response to increasing doses of folate. Notably, the magnitude of de novo DNMT protein expression also followed an inverted U-shaped pattern over the dose range of folic acid treatment.

\section{Therapeutic implications and future perspectives}

Clinically, the central message of the current study by Iskandar et al. is the hope that modification of the methylation milieu may aid in the therapy of the injured CNS via an epigenetic mechanism (7). As a corollary, the authors show that cellular damage begets methyl-group deficiency, so the cell responding to an injury is no longer able to maintain its prior level of DNA methylation. Importantly, contrary to the commonly accepted model, DNA methylation as such has recently been shown to be a reversible biological phenomenon (16). In line with these results, $\left[3^{\mathrm{H}}\right]$ methyl group incorporation has been shown to increase in the injured mouse brain after transient brain ischemia (17). Similarly, hypoxia has been demonstrated to cause DNA hypomethylation (18). DNA damage and repair, cell proliferation, and cell cycle reentry of postmitotic cells may all contribute to injury-induced DNA undermethylation. Furthermore, cellular stress and subsequent oxygen radical formation may result in replacement of DNA base guanine with the oxygen radical adduct 8-hydroxyguanine, which has been shown to profoundly alter methylation of adjacent cytosines (19).

Although the focus here is on the role of folate in epigenetic regulation, the complete picture is no doubt considerably more complex. In particular, it should be noted that functional methyl-group deficiency may also exert direct genetic effects. Methyl-group deficiency leads to the depletion of thymidine triphosphate (dTTP) levels, whereas levels of deoxyuridine triphosphate (dUTP) increase (20). Large quantities of dUTP may be incorporated instead of dTTP during S-phase (or DNA repair). Although the resulting A:U mismatches do not produce mutations, they may hamper recognition by DNA-binding proteins and thereby perturb physiological function. Furthermore, low levels of genomic uracil may be generated during DNA repair or directly via deamination of cytosines. The resulting $\mathrm{G}: \mathrm{U}$ mismatches are premutagenic and, if left unrepaired, lead to GC $\rightarrow$ AT transition mutations (21). Importantly, under conditions of reduced SAM availability, DNMTs may catalyze cytosine deamination in double-stranded DNA (22). Along these lines, we have previously demonstrated that prolonged folate deficiency leads to neurodegeneration in the absence of efficient uracil excision repair (23).

The precise cellular targets for the regenerative actions of folic acid also remain to be elucidated. The beneficial effects of folate supplementation may well extend beyond effects on neurons. Equally important, the influence of folate supplementation on the formation of the glial scar (which consists predominantly of reactive astrocytes and proteoglycans and forms after CNS tissue damage, possibly retarding regeneration) deserves further study. Of note, reactive glia may secrete a variety of growth-inhibitory factors that chemically prevent axonal extension. Additionally, folate effects on glial cells within the graft (e.g., Schwann cells) should not be discounted. Importantly, an epigenetic process involving histone deacetylation has recently been shown to participate in myelin repair (24). Finally, it will be important to define specific molecular targets. This may also help to unravel the biochemical basis for the biphasic curve of folic acid dose-dependent DNA methylation and spinal cord regeneration as shown by Iskandar et al. (7).

In conclusion, the safety and simplicity of folate supplementation in conjunction with the strength of the effects observed in the study by Iskandar and colleagues (7) call for clinical investigations of folate supplementation in patients with spinal cord and neurotrauma. Although clinical evidence is still largely lacking, folate may also have a role in the primary prevention and treatment of neurodegenerative disorders such as dementia and stroke.

\section{Acknowledgments}

The authors receive funding from the VolkswagenStiftung (Lichtenberg program), Bundesministerium für Bildung und Forschung (Center for Stroke Research Berlin), Deutsche Forschungsgemeinschaft (ExcellenceCluster NeuroCure and SFB-TR43), and the European Union (ARISE, grant agreement no. Health-F22008-201024 and EuStroke, grant agreement no. Health-F2-2008-202213).

Address correspondence to: Matthias Endres, Klinik und Poliklinik für Neurologie, Charité - Universitätsmedizin Berlin, Charitéplatz 1, D-10117 Berlin, Germany. Phone: 49.30.450.560.102; Fax: 49.30.450.560.932; E-mail: matthias. endres@charite.de.

1. Catoni GL. S-Adenosylmethionine: a new intermediate formed enzymatically from L-methionine and adenosine triphosphate. J Biol Chem. 1953;204(1):403-416.

2. Balaghi M, Wagner C. DNA methylation in folate deficiency: use of $\mathrm{CPG}$ methylase. Biochem Biophys Res Commun. 1993;193(3):1184-1190.

3. Spector R. Micronutrient homeostasis in mammalian brain and cerebrospinal fluid. J Neurochem. 1989;53(6):1667-1674.

4. Ramaekers VT, et al. Autoantibodies to folate receptors in the cerebral folate deficiency syndrome. NEngl J Med. 2005;352(19):1985-1991.

5. Blom HJ, Shaw GM, den Heijer M, Finnell RH. Neural tube defects and folate: case far from closed. Nat Rev Neurosci. 2006;7(9):724-731.

6. Kronenberg G, Colla M, Endres M. Folic acid, neurodegenerative and neuropsychiatric disease. Curr Mol Med. 2009;9(3):315-323.

7. Iskandar BJ, et al. Folate regulation of axonal regeneration in the rodent central nervous system through DNA methylation. J Clin Invest. 2010;120(5):1603-1616.

8. Berger SL, Kouzarides T, Shiekhattar R, Shilatifard A. An operational definition of epigenetics. Genes Dev. 2009;23(7):781-783.

9. Waterland RA, Jirtle RL. Transposable elements: targets for early nutritional effects on epigenetic gene regulation. Mol Cell Biol. 2003;23(15):5293-5300.

10. Jeltsch A. On the enzymatic properties of Dnmt 1 : specificity, processivity, mechanism of linear diffusion and allosteric regulation of the enzyme. Epigenetics. 2006;1(2):63-66.

11. Chahrour $\mathrm{M}$, et al. MeCP2, a key contributor to neurological disease, activates and represses transcription. Science. 2008;320(5880):1224-1229.

12. Okano M, Bell DW, Haber DA, Li E. DNA methyltransferases Dnmt3a and Dnmt3b are essential for de novo methylation and mammalian development. Cell. 1999;99(3):247-257. 
13. Weaver IC, et al. Epigenetic programming by maternal behavior. Nat Neurosci. 2004; 2004;7(8):847-854

14. Kane MA, et al. Influence on immunoreactive folate-binding proteins of extracellular folate concentration in cultured human cells. J Clin Invest. 1988;81(5):1398-1406

15. Liu M, et al. Structure and regulation of the murine reduced folate carrier gene: identification of four noncoding exons and promoters and regulation of dietary folates. J Biol Chem. 2005;280(7):5588-5597.

16. Rachmandani S, Bhattacharya SK, Cervoni N, Szyf M. DNA methylation is a reversible biological phenomenon. Proc Natl Acad Sci U S A.
1999;96(11):6107-6112.

17. Endres M, et al. DNA methyltransferase contributes to delayed ischemic brain injury. J Neurosci. 2000;20(9):3175-81.

18. Chawla RK, Watson WH, Jones DP. Effect of hypoxia on hepatic DNA methylation and tRNA methyltransferase in rat: similarities to effects of methyldeficient diets. J Cell Biochem. 1996;61(1):72-80.

19. Cerda S, Weitzman SA. Influence of oxygen radical injury on DNA methylation. Mutat Res. 1997;386(2):141-152.

20. Goulian M, Bleile B, Tseng BY. Methotrexateinduced misincorporation of uracil into DNA. Proc
Natl Acad Sci U S A. 1980;77(4):1956-1960.

21. Hagen L, et al. Genomic uracil and human disease. Exp Cell Res. 2006;312(14):2666-672.

22. Shen JC, Rideout WM 3rd, Jones PA. High frequency mutagenesis by a DNA methyltransferase. Cell. 1992;71(7):1073-1080.

23. Kronenberg $G$, et al. Folate deficiency induces neurodegeneration and brain dysfunction in mice lacking uracil DNA glycosylase. J Neurosci. 2008;28(28):7219-7230.

24. Shen $S$, et al. Age-dependent epigenetic control of differentiation inhibitors is critical for remyelination efficiency. Nat Neurosci. 2008;11(9):1024-1034.

\title{
p63 and IRF6: brothers in arms against cleft palate
}

\author{
Amel Gritli-Linde
}

Department of Oral Biochemistry, Institute of Odontology, Sahlgrenska Academy, University of Gothenburg, Göteborg, Sweden.

\begin{abstract}
Cleft lip and cleft palate, which can also occur together as cleft lip and palate, are frequent and debilitating congenital malformations, with complex geneses that have both genetic and environmental factors implicated. Mutations in the genes encoding the p53 homolog p 63 and interferon regulatory factor 6 (IRF6) are major causes of cleft lip and cleft palate, but the molecular and cellular mechanisms underlying this have not been clear. However, in this issue of the JCI, Thomason et al. and Moretti et al. independently show that $\mathrm{p} 63$ and IRF6 operate within a regulatory loop to coordinate epithelial proliferation and differentiation during normal palate development. Disruption of this loop as a result of mutations in $p 63$ or IRF6 causes congenital clefting.
\end{abstract}

The developing organism is a master at using simple paradigms to generate complexity. This is typified by the use, at least during early organogenesis, of the same molecular pathways to drive the development of organs and tissues as diverse as the brain, limbs, teeth, and skin. This, however, has a major disadvantage, in that disruption of a specific molecular pathway can lead to concomitant dysfunction of multiple organs, a condition clinically classified as a syndrome.

In humans, mutations in the gene encoding the transcription factor p63, a p53 homolog, cause several autosomal dominant ectodermal dysplasia syndromes (i.e., syndromes deriving from abnormalities in ectodermal structures, such as the hair, teeth, nails, sweat glands, craniofacial structures, and digits), including ectrodactylyectodermal dysplasia-clefting (EEC) and ankyloblepharon-ectodermal dysplasiaclefting (AEC) syndromes. These are both

Conflict of interest: The author has declared that no conflict of interest exists.

Citation for this article: JClin Invest. 2010; 120(5):1386-1389. doi:10.1172/JCI42821. characterized by cleft lip and/or cleft palate $(\mathrm{CL} / \mathrm{CP})$ and defects in ectoderm-derived tissues, such as the epidermis, hair, teeth, and glands (1). Clinically, AEC syndrome differs from EEC syndrome by the near absence of digit malformations and the occurrence of eyelid fusions (ankyloblepharon) and severe skin erosions (1). Similarly, mutations in the gene encoding the transcription factor interferon regulatory factor 6 (IRF6) cause van der Woude syndrome (VWS) and popliteal pterygium syndrome (PPS), both of which are characterized by many ectodermal anomalies, including CL/CP (2). p63 and IRF6 mutations also have been implicated in nonsyndromic forms of CL/CP (i.e., forms of CL/CP in which the lip and/or palate are the only affected tissues) (3).

A first glimpse of the physiological roles of p63 and IRF6 came from studies of mutant mice. p63-null mice lack a stratified epidermis, teeth, hair, and several exocrine glands and display limb truncations as well as CL and CP $(4,5)$. The skin defects are caused by impaired proliferation and differentiation of epidermal keratinocytes (4). Mice either null for Irf6 (6) or homozygous for $R 84 C$ (7), a common IRF6 mutation in individuals with PPS, replicate several anomalies found in patients with VWS and PPS, including skin impairment and the aberrant intraoral epithelial adhesions that lead to CP. The abnormal development of the skin is due to the inability of epidermal keratinocytes to stop proliferating and to undergo terminal differentiation $(6,7)$.

The discovery that mutations in the genes encoding p63 and IRF6 cause human ectodermal dysplasia syndromes triggered a chain reaction of research aimed at unravelling their functions and identifying their targets during normal and abnormal epithelial development (4-8). Yet, their mode of action, regulation, and targets during normal lip and palate development and the mechanisms behind the genesis of CL/CP in both humans and mouse models are still largely unknown. However, in this issue of the JCI, Moretti et al. (9) and Thomason et al. (10) identify an intriguing relationship between p63 and IRF6 during palate development (well illustrated in Figure 5 in ref. 9) by using an arsenal of experimental strategies.

\section{IRF6 is a direct target of p63}

Studies of the functions of $\mathrm{p} 63$ are complicated by the existence of at least six different isoforms. Two separate transcription start sites generate the $\Delta \mathrm{Np} 63$ isoforms and the p53-like TAp63 isoforms. Transcripts of both the TAp 63 and $\Delta \mathrm{Np} 63$ isoforms undergo alternative splicing, generating three subtypes each, designated $\alpha, \beta$, and $\gamma(4)$. Here, the term $p 63$ is adopted to refer to experiments and data that do not dis- 